\author{
MILITARY TECHNICAL COLLEGE \\ CAIRO - EGYPT
}

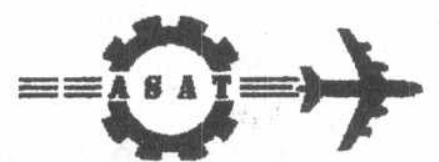

$8^{\text {th }}$ INTERNATIONAL CONF. ON AEROSPACE SCIENCES \&. AVIATION TECHNOLOGY

\title{
ARWAAS Architecture and Implementation Issues
}

\author{
Sameeh. Y. Issa, Matar. A. Matar and A. M. Hamad. \\ Military Technical College \\ Avionics Department
}

\begin{abstract}
The Wide Area Augmentation Systems (WAAS) are being designed and built to provide the required accuracy, availability, integrity and continuity of service to users to so as to reach the satisfaction to rely on global positioning systems as NAVSTAR GPS as the sole means of navigation for all phases of flight in aviation and other maritime as well as land navigation and surveying use. In this paper, a WAAS covering the Arab region from Morocco (west) to Kuwait (east) is proposed; hence dubbed the (ARWAAS). The ARWAAS concept and architecture as well as the layout and interoperability issues are considered.
\end{abstract}

\section{Introduction}

Global positioning systems such as NAVSTAR GPS and GLONASS are being investigated against the specific user requirements, specially the most stringent ones necessitated by the aviation community. This very active area is spawning the Satellite-Based Augmentation Systems(SBAS) to respond to the safety-critical navigation and the specific quality of positioning information required by the aviation community. SBAS are typified by Wide Area Augmentation System (WAAS) in USA, MSAS in Japan and European Geo-stationary Navigation Overlay System (EGNOS). WAAS is, as its name implies, as augmentation to the basic GPS service so as to improve its accuracy, integrity, and availability. Such augmentation system should allow GPS to become a primary means of navigation for en-route travel and non-precision approaches or Category I approaches to airports throughout the region in the coverage area.

Basically, WAAS is based on a connected network of ground reference stations known as (WRS), a processing wide area master station (WMS) and a generally distributing satellite segment to service the user. WRS are implanted in a precisely surveyed point and function to receive GPS signals and determine if any errors exist. Each WRS in the network relays the data to the (WMS) that applies correction algorithms and assesses the integrity of the system. A correction message is prepared and up-linked to the distributing GEO satellite via a ground up-link system (GUS). The message is then broadcast on the a frequency that is required to be the same as GPS (L1,1575.42MHz). Users receive the broadcast information on-board of aircraft flying within the WAAS coverage area. With a navigation payload (as in the INMARSAT-3), GEO communication satellites act as additional navigation 
satellites for the users, thus, improving the availability through providing additional navigation signals for position determination.

Around the world, different groups are currently implementing the wide augmentation system, namely, the European Geo-stationary Navigation Overlay System (EGNOS), the Japanese MTSAT Satellite Augmentation System (MSAS), and the (WAAS) for the U.S.A. [1]. MSAS and WAAS are far from covering the Arab region and EGNOS requires some extension to include the that region [6]. To cover this region and some of the neighbor countries, the ARWAAS (Arabic Wide Area Augmentation System) is a proposed.

Section 2 presents the WAAS concept and architecture. Layout issues of WRS, WMS and satellite transponder are considered in section 3 on the basis of GPS satellite coverage and landing requirements. Section 4 discusses the interoperability issue of ARWAAS with other similar systems. Finally, conclusions are given section 5 .

\section{ARWAAS Concept and Architecture}

The WAAS network includes at least one Station (WMS), a number of Stations (WRS's), arid communication links. Each WRS is equipped with a high-quality clock and a high-quality GPS receiver capable of tracking all satellites within its field of view, a meteorological sensor, a processor, and network hardware for transmission of GPS measurements data to WMS. The WMS solves for the GPS error components based on the known WRS locations and the information collected. The computed error corrections are transmitted to the users via Geostationary Satellite or any convenient communication link.

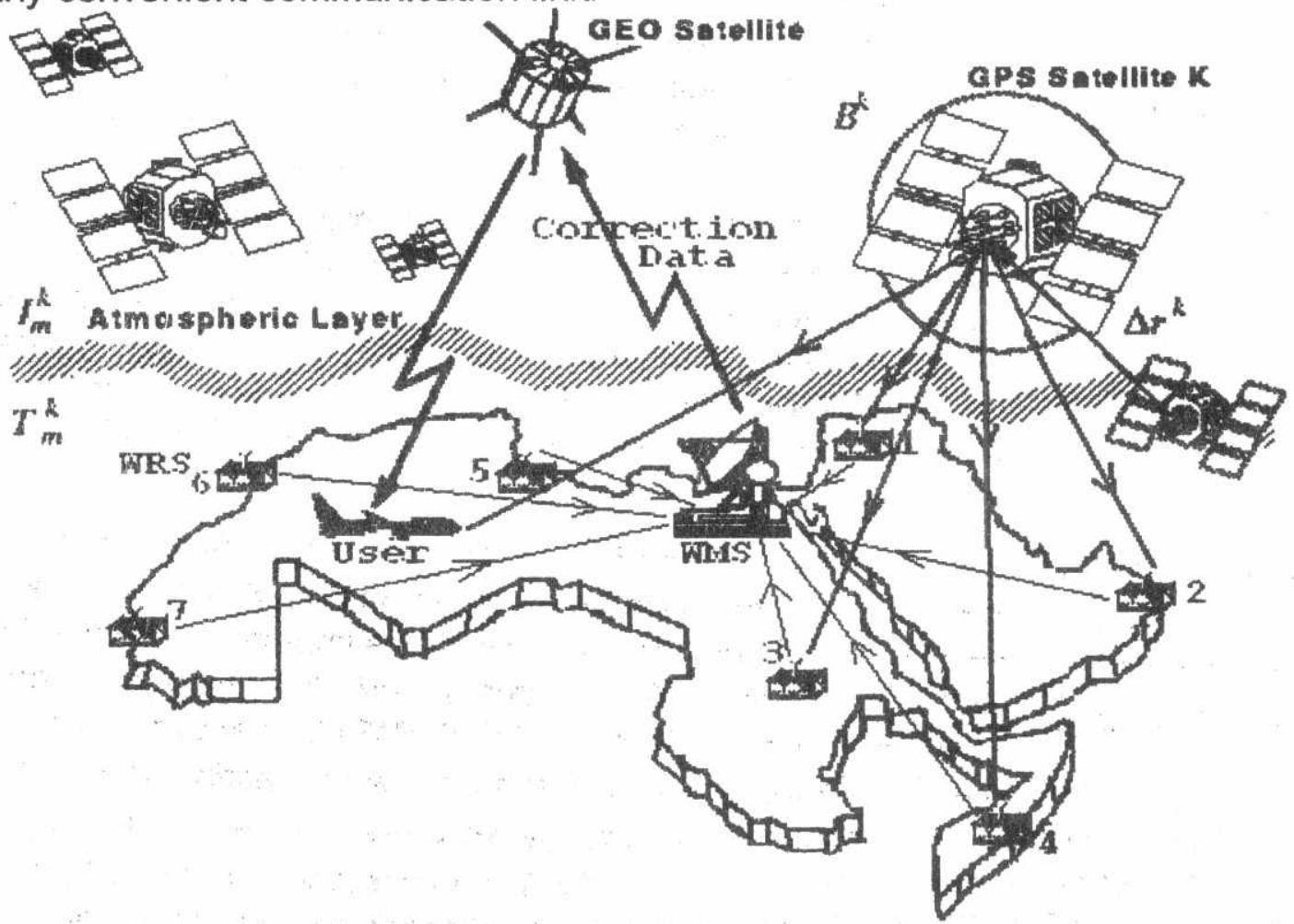

Fig(1) ARWAAS concept. 
Fig. 1 provides an illustration of WAAS and Fig. 2 shows the flow of information between the system components. The process can be summarized as follows:

1) WRS at known locations collect GPS pseudoranges from all satellites in view.

2) Pseudoranges and dual frequency ionospheric delay measurements (if available) are sent to the WMS.

3) WMS computes an error correction vector.

4) Error correction vector is transmitted to users.

5) Users apply error corrections to their measured pseudoranges and collected ephemeris data to improve navigation accuracy.

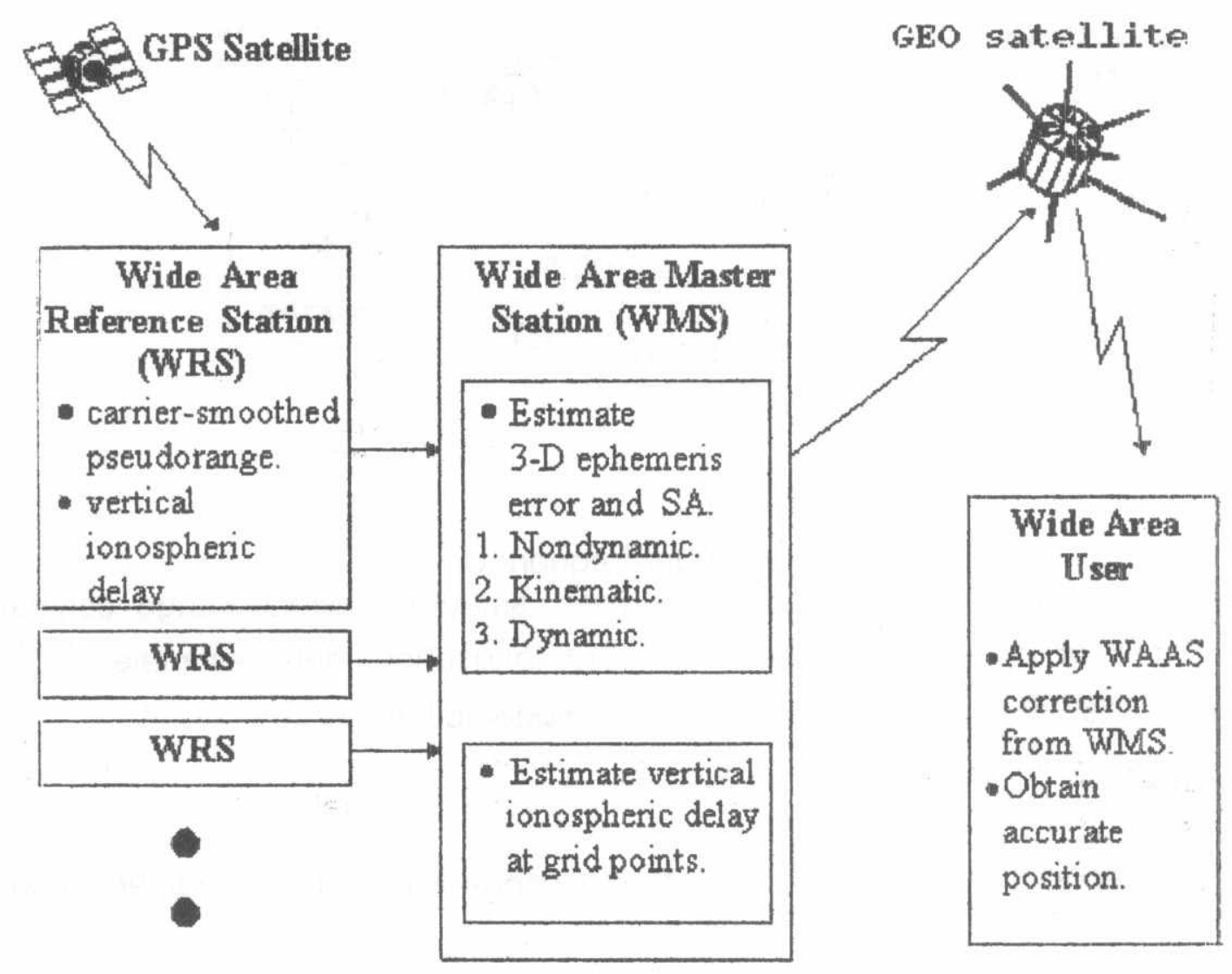

Fig(2) Block diagram of ARWAAS components.

\subsection{The Wide Area Reference Station (WRS)}

WRS's are distributed throughout the area where service is to be provided. Each WRS comprises three independent pieces of equipment, consisting of an antenna, GPS receiver, processor, power conditioner, and frequency standard, in order to provide redundancy and overall system integrity. There are two types of the WRS's: 1-WRS with dual-frequency GPS receiver.

2-WRS with single-frequency GPS receiver. 


\subsubsection{WRS with dual-frequency GPS receiver}

This WRS uses a dual frequency receiver to acquire code observation at the GPS frequencies $f_{\mathrm{L} 1}$ and $f_{\mathrm{L} 2}$. (see Fig .3). The set of code observations at $f_{\mathrm{L} 1}$ and $f_{\mathrm{L} 2}$ yield $\left\{\rho_{m, L 1}^{(k)}\right\}_{k=1}^{K_{m}}$ and $\left\{\rho_{m, L 2}^{(k)}\right\}_{k=1}^{K_{m}}$ respectively, where $K_{m}$ is the number of satellites in view of the $m^{\text {th }}$ reference station.

$$
\begin{aligned}
& \rho_{m, L_{1}}^{(k)}=\left(r_{m}^{(k)}+b_{m}-B^{(k)}\right)+I_{m, L_{1}}^{(k)}+T_{m}^{(k)}+v_{m, \tau}^{(k)} \\
& \rho_{m, L_{2}}^{(k)}=\left(r_{m}^{(k)}+b_{m}-B^{(k)}\right)+I_{m, L_{2}}^{(k)}+T_{m}^{(k)}+v_{m, \tau}^{(k)} .
\end{aligned}
$$

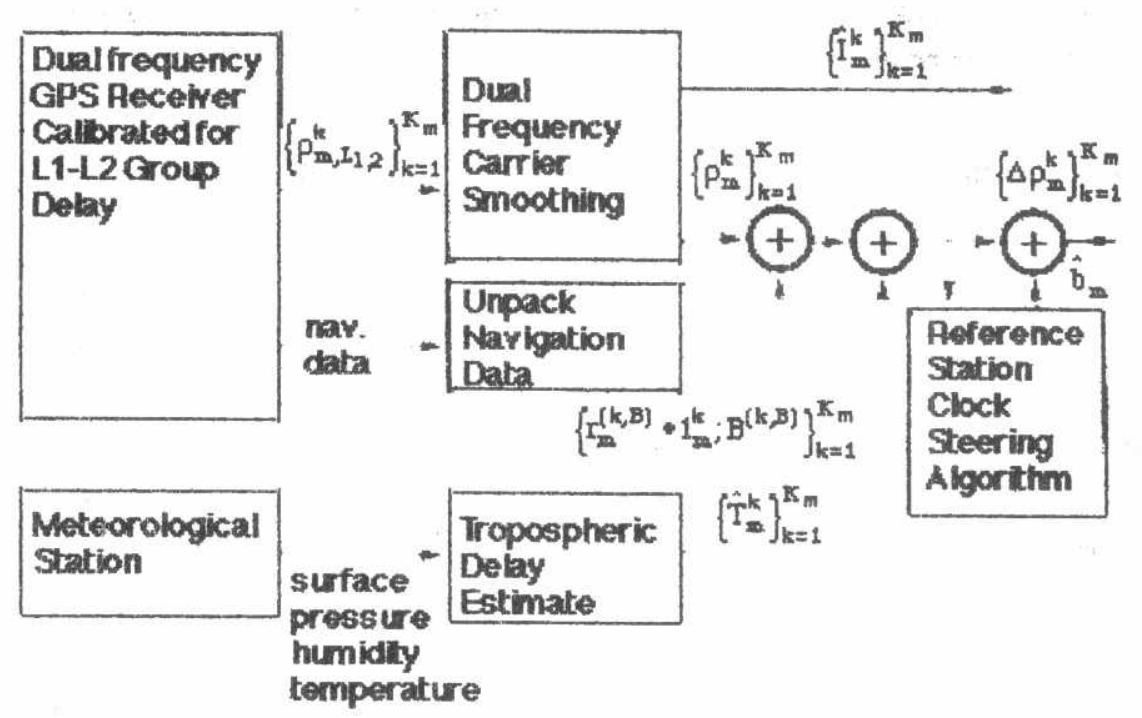

Fig(3) WRS with dual-frequency.

The carrier smoothing algorithm delivers smoothed pseudorange estimates $\left\{\rho_{m}^{k}\right\}_{k=1}^{K_{m}}$ free of ionospheric delays and the ionospheric delay estimates $\left\{I_{m}^{k}\right\}_{k=1}^{K_{m}}$. These smoothed pseudorange estimates are modeled by:

$$
\begin{aligned}
\rho_{m}^{(k)} & =\left(r_{m}^{(k)}+b_{m}-B^{(k)}\right)+\Delta M_{m}^{(k)}+T_{m}^{(k)}+v_{m, \tau}^{(k)} . \\
& =\left(r_{m}^{(k)}+b_{m}-B^{(k)}\right)+T_{m}^{(k)}+v_{m, \tau}^{(k)} .
\end{aligned}
$$

where the second line assumes that the ionospheric error term is too small to be included in the noise term.

Computed from the satellite data in the navigation message, the pseudorange and the nominal range to the satellite (known location of the WRS) are compared to get the required correction at the WRS site. The satellite clock offset (described by the clock field in the navigation message) is also taken into account. Thus pseudorange errors reduce to:

$$
\begin{aligned}
\Delta \rho_{m}^{(k)} & =\left|r_{m}^{(k)}\right|-\left|r_{m}^{(k, B)}\right|+b_{m}-\left(B^{(k)}-B^{(k, B)}\right)+T_{m}^{(k)}+v_{m, \tau}^{(k)} \\
& =\Delta r^{k} \cdot 1_{m}^{k}+b_{m}-\left(B^{k}-B^{k, B}\right)+T_{m}^{k}+v_{m, \tau}^{k} \\
& =\Delta r^{k} \cdot 1_{m}^{k}+b_{m}-\Delta B^{k}+T_{m}^{k}+v_{m, \tau}^{k}
\end{aligned}
$$

where 
$\Delta r^{k}$ is the vector relating the true location of the satellite, $r^{k}$, and the location given by the navigation message, $r^{k, B}$.

$1_{m}^{k}$ denotes the unit vector from the $k^{\text {th }}$ satellite towards the $m^{\text {th }}$ WRS. $B^{k}$ is the true offset of the satellite transmission from GPS time.

$B^{\mathrm{k}, \mathrm{B}}$ is the offset according to the navigation message.

WRS estimates the tropospheric delay on the basis of the elevation angle and local measurements of pressure, temperature and humidity. Indeed, a model without this auxiliary information can suffer errors of 2 meters for satellites at low elevation angles. In contrast, a model with the surface measurements does not usually have errors greater than 30 centimeters.

Finally, the WRS uses a "clock steering" algorithm to estimate the WRS clock offset. This algorithm averages the reduced pseudorange from all of the satellites in view to form an estimate of the WRS clock. This averaging reduces the erroneous contribution due to the individual satellite clock and ephemeris errors as well as the pseudorange measurement noise. In addition, if the WRS has a high quality clock such as a Rubidium oscillator, then the WRS clock estimates can be averaged over time to further reduce the clock estimate error. This averaging strategy yields pseudorange error estimates that are approximate but physically meaningful the WRS. However, it may exhibit jumps in the clock estimates as the set of satellites in view changes. and must be taken into account.

The WRS clock estimates are subtracted from the smoothed pseudoranges to yield:

$$
\Delta \rho_{m}^{k}=\Delta r^{k} \cdot 1_{m}^{k}+\Delta b_{m}-\Delta B^{k}+T_{m}^{k}+v_{m, \tau}^{k}
$$

As shown in Fig.3, these pseudorange residuals are sent along with the vertical ionospheric delay estimates, $\left\{I_{m}^{k}\right\}_{k=1}^{K_{m}}$, to the WMS for further processing.

\subsubsection{WRS with single-frequency GPS receiver}

In this case WRS uses a single frequency receiver to acquire code observation at the GPS frequencies $f_{\mathrm{L} 1}$ to yield $\left\{\rho_{m, L 1}^{(k)}\right\}_{k=1}^{K_{m}}$.

The WRS uses coefficients of the ionospheric delay which are transmitted within the navigation message to calculate the ionospheric delay model. This model will eliminate $(50-60) \%$ from the ionospheric delay.

All other processes are the same as the WRS with dual frequency receivers.

\subsection{Wide Area Master Stations (WMS):}

Using WRS's pseudorange residuals along with the vertical ionospheric delay estimates $\left\{I_{m}^{k}\right\}_{k=1}^{K_{m}}$, the WMS combines the relevant satellite information to yield the correction to be adhered to a given satellite data.

The WMS incorporates two main estimators (see Fig.4):

1- The orbit and clock error estimator.

2- The ionospheric delay estimator. 
The first, uses the iono- and tropo-free pseudorange residuals from the WRS's to estimate the clock and location errors for each satellite in view of the network[ 2,3 ]. The second, uses the ionospheric delay data to generate ionospheric delay estimates for grid of locations which uniformly cover the service area[ 2,3 ].

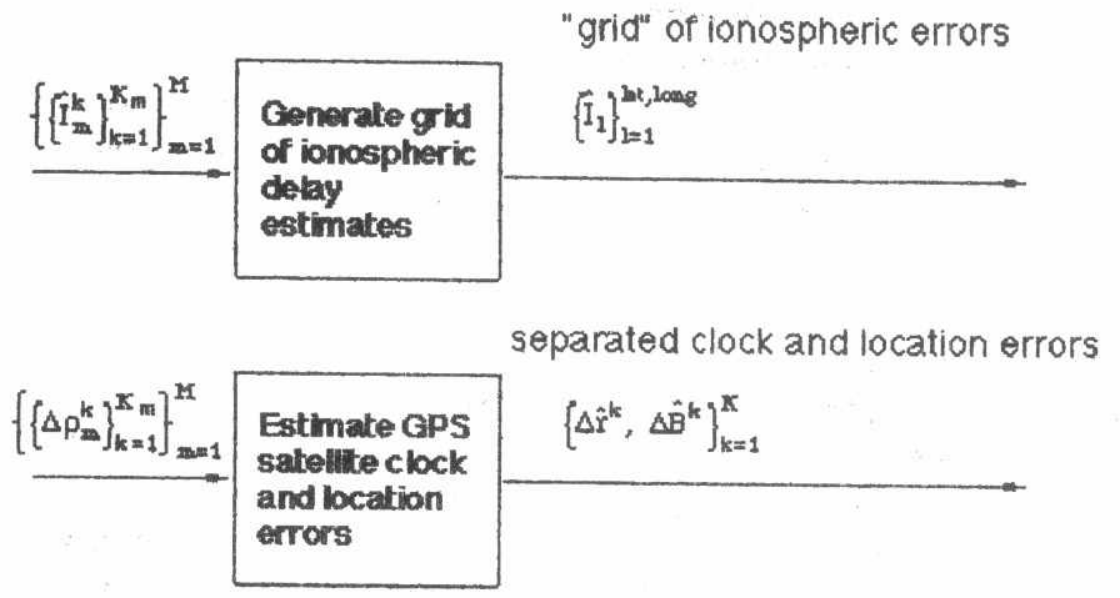

Fig(4) WMS Estimators.

\subsection{The Geostationary Satellite:}

In order to send the correction data from the WMS to the user, we can use any geostationary satellite (communication satellite). This satellite will be send the GPS integrity with correction data to GPS users and may also provide a ranging signal that augments GPS. Ranging information is to be transmitted at $L_{1}$ frequency.

For a "general "frequency selection of the ARWAAS receiver, three options: Arabsat, Nilesat, or inmarsat-3 are considered. Selection is subject to the RTCA standards[1,2,5] for SBAS and to receiver configuration as well as frequency assignment to such application and is being currently investigated.

\subsection{The User Segment}

The user equipment combines pseudoranges and ephemeris parameters for GPS satellite in view with error correction vector sent by the WMS via a geostationary satellite link. A filter is to be used to reduce the receiver noise and the multipath errors and to eliminate the ionospheric time delays. Black's model $[1,5]$ is used to reduce the tropospheric errors as done in WRS monitor stations. The error correction are applied to the measurements, already adjusted for the atmospheric errors to form the solution for the user's position and clock bias. User receivers will be either a combination of the conventional GPS with another receiver for the ARWAAS signals of an upgraded GPS receiver if the ARWAAS signals including the ranging signal are at the $L 1$ frequency. Very active market is challenging for the definition and promotion of such a receiver. 


\section{The Ground Segment (WRS + WMS) Layout Issues}

To implement the (ARWAAS), it is required to:

- Select the number and location of WRS s.

- Select the number and location of the WMS.

- Design the processing algorithms for data in the WMS.

- Select the Geo-stationary satellite and the interconnection network.

- Format and distribute data from WMS.

- IJpgrade the user equipment to accept data of ARWAAS.

As to the layout of WRS, we solve this problem on the basis of the GPS satellite coverage and landing requirements

\subsection{GPS Satellite Coverage}

The geometry of satellite coverage of the earth is shown in Fig.5. A satellite at an altitude $h$ above the navigator covers a spherical segment $\beta$ that depends on the mask angle $\alpha$ or minimum angle of visibility.

Note that $\alpha$ is the minimum elevation of the satellite above the horizon that allows the navigator to establish a fix.

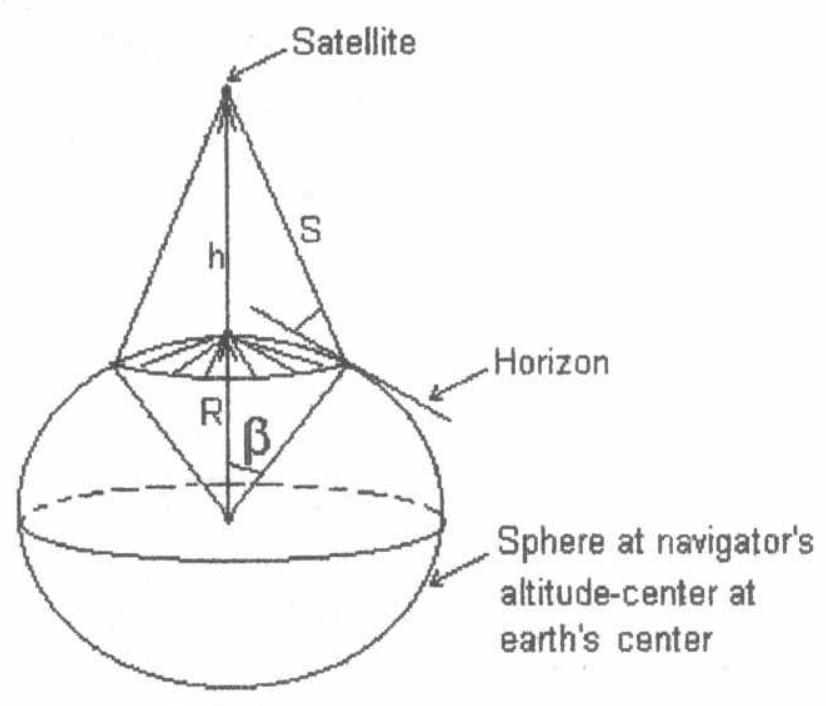

Fig(5) Area covered by single navigation satellite.

The arigle $\beta$ (observed central angle) is measured by the great-circle arc from the satellite sub-satellite point to the circle bounding the area covered by the satellite. By the law of cosines[4]:

$$
h=R\left[\frac{\cos (\alpha)}{\cos (\alpha+\beta)}-1\right]
$$

yielding the observed half-arc angle as:

with :

$$
\beta=\cos ^{-1}\left[\frac{R \cos (\alpha)}{R+h}\right]-\alpha
$$

$\mathrm{R}$ Radius of navigator(equals earth radius + navigator altitude). 
h Satellite altitude above navigator and S Maximum slant range(see Fig.5).

Geometrical relation between the observed half-arc $\beta$, satellite altitude $h$, and minimum elevation angle $\alpha$ is evaluated and plotted in Fig.6 to determine the satellite coverage versus satellite altitude. Coverage is represented by the arc length of coverage (R. $\beta$ ) at the earth's surface (i.e, navigator's altitude is zero).

At satellite altitude of $18520 \mathrm{~km}$, nearly $35 \%$ of the earth surface is covered.

The radius of coverage at GPS height $(20000 \mathrm{~km})$ is about $4000 \mathrm{~km}$; determined only by the height of the orbit and the mask angle. Taking into account the power of the transmitters, the size and characteristics of the antennas used in the satellite and at the navigator's station the coverage area will be reduced. However, over about $30 \%$ of the Earth surface, the minimum number of stations required to determine the satellite orbit are 3 stations taking several snapshots during the period from "satellite rise" to " satellite set" ranging to more than 2 hours.

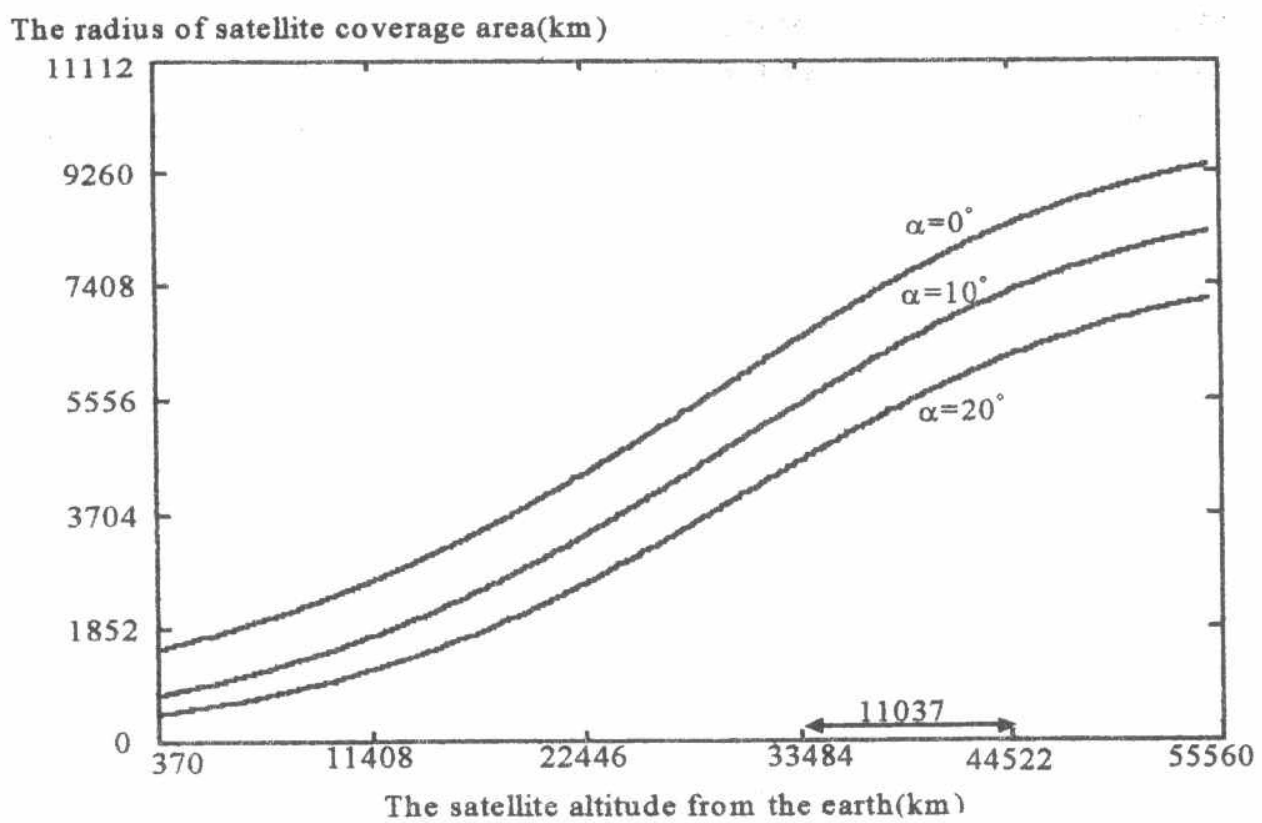

Fig(6) The satellite coverage area versus satellite altitude

Knowing that the distance between the Rabat and Masqat is about $6500 \mathrm{~km}$, and that the Arab region is covered by three GPS satellite orbits implying that a minimum number of WRS s required for the ARWAAS solution will be 7 monitoring stations for improved reliability of the solution taking into account the reuse of these stations for other satellites. The layout of these 7 stations will be given in the next sub-section.

\subsection{AC landing Requirements}

For precision landing(CAT 3) on the basis of GPS, it is required to provide the Local Area Augmentation System (LAAS) service in the main airports. These LAAS Base Stations will be used for a dual function; i.e. as WRS's for the ARWAAS to enhance the accuracy and reliability of the solution. This results in more than 20 WRS used to form the ARWAAS reference stations set. 
Proposing Cairo as WMS and the minimum seven WRS's are given in Table.1 with its geographical position(longitude and latitude ).

\begin{tabular}{|c|c|c|c|c|c|}
\hline NO & $\begin{array}{l}\text { STATION } \\
\text { LOCATION }\end{array}$ & $\begin{array}{l}\text { THE } \\
\text { TASK }\end{array}$ & $\begin{array}{l}\text { THE STATION } \\
\text { COUNTRY }\end{array}$ & $\begin{array}{l}\text { LONGITUDE } \\
\text { (DEGREE) }\end{array}$ & $\begin{array}{l}\text { LATITUDE } \\
\text { (DEGREE) }\end{array}$ \\
\hline 1 & CAIRO & WMS & EGYPT & $3116 \mathrm{E}$ & $304 \mathrm{~N}$ \\
\hline 2 & DAMASCUS & WRS & SYRIA & $36 \quad 15 \mathrm{E}$ & $3333 \mathrm{~N}$ \\
\hline 3 & MASQAT & WRS & OMAN & $58 \quad 33 \mathrm{E}$ & $2338 \mathrm{~N}$ \\
\hline 4 & Mogadishu & WRS & SOMALIA & $4523 \mathrm{E}$ & $26 \mathrm{~N}$ \\
\hline 5 & KHARTOUM & WRS & SUDAN & $3229 \mathrm{E}$ & $1534 \mathrm{~N}$ \\
\hline 6 & TRIPOLI & WRS & LIBYA & $1310 \mathrm{E}$ & $3252 \mathrm{~N}$ \\
\hline 7 & RABAT & WRS & MOROCCO & $652 \mathrm{~W}$ & $341 \mathrm{~N}$ \\
\hline 8 & Nouakchott & WRS & MAURITANIA & $1559 \mathrm{~W}$ & $184 \mathrm{~N}$ \\
\hline
\end{tabular}

Table (1) The WRS position in the ARWAAS.

\subsection{Utilization of Percolated Stations ( SKYFIX and EGNOS)as WRS's}

Several stations have been located for maritime services like the SKYFIX network distributed over Europe, Middle East and Africa. They can be used to function as reference stations, EGNOS as SBAS covers Europe, and can be extended [6] to include some of the Middle East and Africa and its WRS can be shared with ARWAAS network apart from the interoperability issue discussed in the next section.

\section{ARWAAS -to- SBAS Interoperability Issue}

Currently developed SBAS systems are EGNOS, MSAS and WAAS in addition to the proposed ARWAAS system.

MSAS has a total of 8 ground reference stations, six of them are in Japan itself, one in Hawaii and another in Australia. The service area is too far to cover the Middle East zone. South and North America WAAS has 24 reference stations located across the United States and Canada. These reference stations have all been installed and tested in anticipation of Phase I WAAS being operational in mid- 2000 . As in case of MSAS, this WAAS service area is too far to cover the Middle East zone.

EGNOS -with its 44 stations- represents the largest geographical distribution of all of the developing SBAS system. Most of the stations are located in Europe with periphery stations located in Africa. This system is the most likely one to be extended [6] to our region and its coverage area is intersecting with the proposed ARWAAS.

These currently developed SBAS should provide coverage within their service volumes but as users pass from one SBAS to another there will likely be a disruption in coverage since none of current systems coverage area overlap. To reach seamless coverage between various SBAS systems, the interoperability should be secured .A byproduct of satisfying this primary goal of interoperability, is the incorporation of data over a wider geographical region and consequently increasing the strength of each individual SBAS and improving the performance for user on the periphery of SBAS coverage. 
If a user were to operate between these SBAS systems then one strategy for integration would be to switch from the departure SBAS to the arrival SBAS en-route without sharing any information either explicitly in the user avionics or implicitly through ground infrastructure. This switching would occur at the location where the number of corrections for the arrival SBAS, on average, exceeds that of the departure SBAS. Another interoperability strategy is based upon sharing the resources on either of the subsequent bases.

The interoperation can take place at three distinct levels in the SBAS [5]:

1) Reference Data/State Information.

2) Avionics Integration.

3) Shared Satellite.

These issues are under consideration for ARWAAS , specially with EGNOS.

\section{Conclusion}

A wide area augmentation system for the Arab region dubbed AR WAAS is considered. A set of at least 7 WRS stations are required and their layout issues are introduced. Location of each of these WRS is precisely surveyed, enabling to determine any error in the GPS signals being received at its own location. This information is then passed through a communication network to a WMS proposed to be located in Egypt. The correction data calculated at WMS is then put to the user through a geo-stationary communications satellite.

For dual use in ARWAAS and LAAS services additional WRS in the important Arabic airports are to be implanted. This would yield more accurate and reliable wide area system useable for aircraft receivers, maritime as well as for ground systems applications. Detailed investigation of the proposed configuration is currently being done as a part of Ph.D thesis. The orbit and clock estimation for ARWAAS is given in a companion paper.

\section{References}

[1] E.D. Kaplan, 2Ed. "Understanding GPS: Principles and Applications", Chap.7,8 published by Artech House 1997.

[2] C. Kee, T. Walter, Y.C. Chao, Y.J. Tsai, P. Enge and B. W. Parkinson," Comparison of Master Station and User Algorithms for WAAS“ , Journal of Guidance, Control, and Dynamics, V 20, No 1, P.P 170-176, February 1997.

[3] P. Enge, T. Walter, S. Pullen, and C. Kee," Wide Area Augmentation of the Global Positioning System", Proceedings of the IEEE, Vol. 84, No. 8, August 1996.

[4] M. Kayton and W.R.Fried,Ed. "Avionics Navigation Systems", Chap.12 Published by J.Wiley \& Sons, $1^{\text {st }}$ edition, 1969.

[5] R. Fuller, D. Dai, T. Walter, and P. Enge, "Interoperation and integration of Satellite Based Augmentation System", Proceedings of ION GPS 98, Institute of Navigation, 1998.

[6] H.EI-Saadawy."The Extension of EGNOS Project to Cover The Southern Coast of the Mediterranean Sea and the Middle East " Proc. GNSS 98,pp.II-O1.7 vol.1, Toulouse ,France, October 1998. 